www.nature.com/jhg

\title{
A family of oculofaciocardiodental syndrome (OFCD) with a novel BCOR mutation and genomic rearrangements involving $\mathrm{NHS}$
}

\begin{abstract}
Yukiko Kondo $^{1}$, Hirotomo Saitsu ${ }^{1}$, Toshinobu Miyamoto ${ }^{2}$, Kiyomi Nishiyama ${ }^{1}$, Yoshinori Tsurusaki ${ }^{1}$, Hiroshi Doi ${ }^{1}$, Noriko Miyake ${ }^{1}$, Na-Kyung Ryoo ${ }^{3}$, Jeong Hun Kim ${ }^{3}$, Young Suk $\mathrm{Yu}^{3}$ and Naomichi Matsumoto ${ }^{1}$

Oculofaciocardiodental syndrome (OFCD) is an X-linked dominant disorder associated with male lethality, presenting with congenital cataract, dysmorphic face, dental abnormalities and septal heart defects. Mutations in BCOR (encoding BCL-6interacting corepressor) cause OFCD. Here, we report on a Korean family with common features of OFCD including bilateral 2nd-3rd toe syndactyly and septal heart defects in three affected females (mother and two daughters). Through the mutation screening and copy number analysis using genomic microarray, we identified a novel heterozygous mutation, $c .888 \mathrm{delG}$, in the $B C O R$ gene and two interstitial microduplications at $\mathrm{Xp22.2-22.13}$ and $\mathrm{Xp21.3}$ in all the three affected females. The $B C O R$ mutation may lead to a premature stop codon (p.N297IfsX80). The duplication at Xp22.2-22.13 involved the NHS gene causative for Nance-Horan syndrome, which is an X-linked disorder showing similar clinical features with OFCD in affected males, and in carrier females with milder presentation. Considering the presence of bilateral 2 nd-3rd toe syndactyly and septal heart defects, which is unique to OFCD, the mutation in BCOR is likely to be the major determinant for the phenotypes in this family.
\end{abstract}

Journal of Human Genetics (2012) 57, 197-201; doi:10.1038/jhg.2012.4; published online 2 February 2012

Keywords: BCOR; congenital cataract; frameshift mutation; genomic rearrangement; Nance-Horan syndrome; NHS; oculofaciocardiodental syndrome

\section{INTRODUCTION}

Oculofaciocardiodental syndrome (OFCD, Mendelian Inheritance in Man (MIM) \#300166), an X-linked dominant disorder, is characterized by ocular, facial, cardiac and dental abnormalities associated with male lethality. ${ }^{1,2}$ Mutations in the BCL-6 corepressor gene $(B C O R$, $\left.\mathrm{MIM}{ }^{*} 300485\right)$ at Xp11.4 cause OFCD. ${ }^{3}$ BCOR/Bcor is ubiquitously expressed in human tissues and is strongly and specifically expressed in the eye, brain, neural tube and branchial arches during mouse embryonic development, which are affected in OFCD. ${ }^{4,5}$ In 2009, Hilton et al. ${ }^{6}$ clinically reviewed 35 cases with BCOR mutations and summarized the frequency of phenotypes: congenital cataract (100\%), microphthalmia and/or microcornea (82\%), facial dysmorphism (96\%) including long narrow face and high nasal bridge, cardiac anomalies (74\%, commonly septal defects), dental abnormalities $(100 \%)$ such as delayed and/or primary dentition, root radiculomegaly, and absent/duplicated/fused teeth and mental retardation (18\%). ${ }^{6}$ Additionally, skeletal abnormalities such as 2 nd-3rd toe syndactyly, hammer toes, and radioulnar synostosis are also observed in $97 \%$ patients. Various types of mutations in BCOR have been described including nonsense, small insertions or deletions and splice site mutations, suggesting that the loss of functions (null allele) might result in OFCD. In addition, microdeletions involving $B C O R$ have been also reported in individuals with OFCD. Most mutations predicted to generate premature stop codons, likely suffering from nonsense-mediated mRNA decay, although nonsense-mediated mRNA decay was unable to be confirmed because of the severe skewed $\mathrm{X}$-inactivation in blood leukocytes. ${ }^{3,6}$

Nance-Horan syndrome (NHS) is an X-linked cataract-dental syndrome (MIM \#302350) characterized by congenital cataract, dental abnormalities, facial dysmorphism and mental retardation. ${ }^{7}$ Congenital cataract in affected male usually requires early surgery. ${ }^{8}$ Dental abnormalities include maxillary and mandibular diastema of both central and lateral incisors, and screwdriver-shaped teeth because of narrow gingival and incisal margins. ${ }^{9}$ Carrier females typically display posterior Y-sutural lens opacities, and the dental and facial anomalies of the syndrome may be observed, but with a milder presentation. ${ }^{8}$ Mutations in NHS (MIM $\left.{ }^{*} 300457\right)$ at Xp21.1-p22.3 cause NHS. ${ }^{9-11}$ The most pathogenic mutations are truncating mutations. Coccia et al. ${ }^{8}$ reported complex duplication-triplication rearrangements of the NHS gene in a family with congenital cataract and congenital

\footnotetext{
${ }^{1}$ Department of Human Genetics, Yokohama City University Graduate School of Medicine, Yokohama, Japan; ${ }^{2}$ Department of Obstetrics and Gynecology, Asahikawa Medical College, Asahikawa, Japan and ${ }^{3}$ Department of Ophthalmology, Seoul National University College of Medicine, Seoul, Korea

Correspondence: Dr N Matsumoto, Department of Human Genetics, Yokohama City University Graduate School of Medicine, Fukuura 3-9, Kanazawa-ku, Yokohama 236-0004, Japan.

E-mail: naomat@yokohama-cu.ac.jp

Received 3 October 2011; revised 6 December 2011; accepted 5 January 2012; published online 2 February 2012
} 
heart defects in affected males, suggesting that genomic rearrangements of NHS are able to cause the X-linked cataract.

In this report, mutation screening and genomic microarray revealed a heterozygous mutation in $B C O R$ and genomic rearrangements involving NHS in the three affected females of a Korean family with congenital cataract, dental abnormalities and 2nd-3rd toe syndactyly. Detailed molecular analysis will be presented.

\section{MATERIALS AND METHODS}

\section{Clinical report}

The Korean family with congenital cataract was previously described (as family 4) (Figure 1a). ${ }^{12}$ Clinical features are summarized in Table 1. In the elder sister (MC17, the proband), bilateral congenital cataracts were noted 100 days after birth. Bilateral lensectomy and secondary intraocular lens insertion were performed. Ventricular septal defect, atrial septal defect, patent ductus arteriosus, delayed dentition, bilateral broad halluces, bilateral 2nd-3rd toe syndactyly, bilateral hammer toes and right brachyphalangia of fourth toe were also recognized. Mental development was normal. In the younger sister (MC18), bilateral congenital cataracts were also recognized. Bilateral lensectomy and secondary intraocular lens insertion were performed at ages of 2 months and 3 years, respectively. Right inguinal hernia, delayed dentition, and bilateral broad halluces, bilateral 2nd-3rd toe syndactyly, and bilateral hammer toes were noted (Figures $1 \mathrm{~b}$ and $\mathrm{c}$ ). She had learning difficulties at school, but IQ was not measured. In the mother (MC17b), bilateral congenital cataracts were noted and left lensectomy was performed at age of 10 years. Because of her dental anomalies and hypodontia, all her teeth were surgically removed. Bilateral 2nd-3rd toe syndactyly and bilateral hammer toes were noted. Her intelligence was normal. All the three affected members shared bilateral congenital cataracts, delayed dentition, bilateral 2nd-3rd toe syndactyly and bilateral hammer toes. Dysmorphic facial features were unseen.

\section{DNA sequencing}

Experimental protocols were approved by Institutional Review Boards for Ethical Issues at Yokohama City University School of Medicine and the Committee for the Ethical Issues on Human Genome and Gene Analysis, Seoul National University. Informed consent was obtained from all individuals. Genomic DNA was obtained from peripheral leukocytes using QIAGEN Blood and Cell Culture DNA Midi Kit (QIAGEN, Hilden, Germany). DNA was amplified using GenomiPhi V2 kit (GE healthcare, Buckinghamshire, UK). In $B C O R$, there are three isoforms: isoform a (GenBank accession number NM_017745.5), isoform b (GenBank accession number NM_001123384.1) and isoform c (GenBank accession number NM_001123385.1). In NHS, there are two isoforms: isoform 1 (GenBank accession number NM_198270.2) and isoform 2 (GenBank accession number NM_001136024.2). Nucleotide sequences of 1st to 15th exons of BCOR and 1st to 8th exons of NHS covering all the protein coding region as well as exon-intron borders were analyzed. Polymerase chain reaction (PCR) conditions and primer information are shown in Supplementary Table 1. PCR products were purified with ExoSAP (USB, Cleveland, OH, USA) and sequenced with BigDye terminator 3.1 (Applied Biosystems, Foster City, CA, USA) on 3100 and $3500 \times 1$ Genetic Analyzer (Applied Biosystems). Sequences of patients were compared with the reference human genome sequences (based on the UCSC Genome Browser coordinate, February 2009) with Sequencher 4.10.1. (Gene Codes, Ann Arbor, MI, USA).

\section{Copy number analysis}

Copy number variations (CNVs) were investigated by Cytogenetics WholeGenome 2.7 M. Array (Affymetrix, Santa Clara, CA, USA) based on the manufacturer's instruction using $100 \mathrm{ng}$ genomic DNA from three affected females (MC17b, MC17 and MC18). Copy number alterations were analyzed by Chromosome Analysis Suite (Affymetrix) with NetAffx 30.1 annotations (hg18 assembly). Any filters such as minimum size and probe numbers of CNVs were not applied. The selection criteria for putative pathogenic CNVs were as follows: (1) CNVs were shared with three affected females, (2) CNVs a
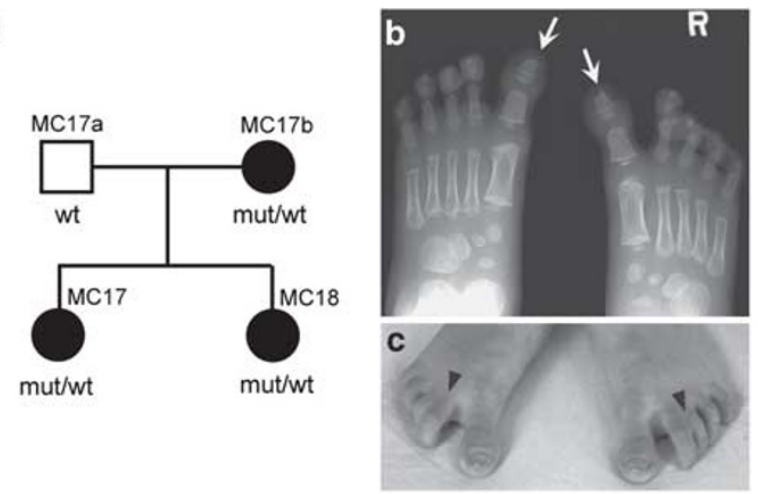

d
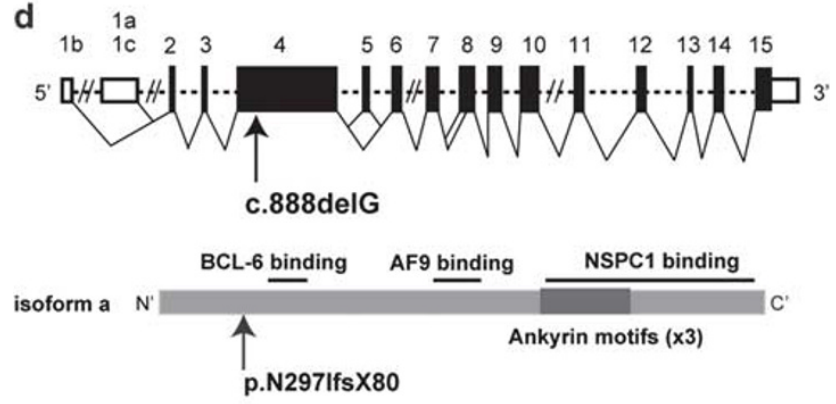

e

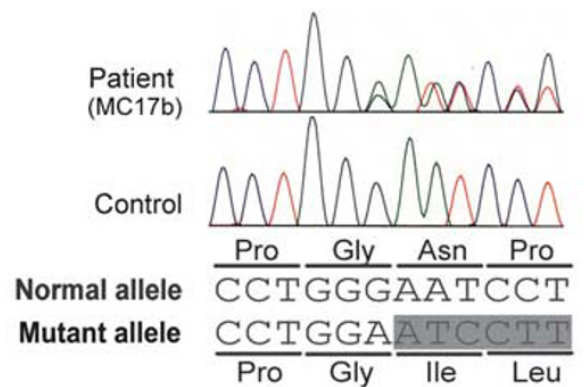

Figure 1 Pedigree, foot malformation and a $B C O R$ mutation found in the family. (a) Familial pedigree. Black and open symbols denote affected and unaffected individuals, respectively. (b, c) Bilateral broad halluces (the big toe, arrows in b), bilateral 2nd-3rd cutaneous syndactyly (arrowheads in $\mathbf{c}$ ) and bilateral hammer toes in $\mathrm{MC18}$. (d) Schematic representation of the $B C O R$ gene (top). UTR and coding region are open and filled rectangles, respectively. Alternative splicing by three different isoforms is shown. The isoform b is absence of exon 5 and the isoform c is $102 \mathrm{bp}$ and $156 \mathrm{bp}$ longer than the isoform $a$ and $b$, respectively. The location of the $c .888 \mathrm{del} G$ mutation is indicated by an arrow. The protein structure of BCOR (isoform a, bottom). Three consecutive ankyrin motifs are indicated as a dark-gray box. The three binding sites for BCL-6, AF9 and NSPC1 are indicated with horizontal bars. (e) Electropherograms showing the mutation in the affected patient (MC17b) (top) and a control (bottom). A single nucleotide deletion in exon 4 results in a frameshift. mut, a mutant allele; wt, a wild type allele.

encompassed exons and (3) CNVs were not present in the Database of Genomic Variants (http://projects.tcag.ca/variation/).

\section{Cloning of duplication breakpoints}

DNA of the affected mother (MC17b) was digested with restriction enzymes: EcoRI, NsiI, XbaI, BamHI and BglII (New England Biolabs, Beverly, MA, USA). Digested DNA was self-ligated by Ligation High ver. 2 (Toyobo, Osaka, Japan), precipitated with ethanol, and dissolved in $20 \mu \mathrm{l} \mathrm{EB}$ buffer (QIAGEN, Tokyo, Japan). Inverse PCR was performed in $25 \mu \mathrm{l}$ volume containing $2 \mu \mathrm{l}$ ligated DNA, $1 \times$ PCR buffer for KOD FX, 0.4 mM each dNTPs, $0.5 \mu \mathrm{M}$ each primer and 
Table 1 Clinical features of patients with a BCOR mutation

\begin{tabular}{|c|c|c|c|}
\hline & $M C 17 b$ & MC17 & MC18 \\
\hline Age & 41 & 11 & 8 \\
\hline Sex & Female & Female & Female \\
\hline \multicolumn{4}{|l|}{ Ocular features } \\
\hline Congenital cataract & + & + & + \\
\hline Microphthalmia/microcornea & - & - & - \\
\hline Coloboma & - & - & - \\
\hline Ptosis & - & - & - \\
\hline Secondary glaucoma & - & + & + \\
\hline Nystagmus & - & - & - \\
\hline Cardiac features & - & + & - \\
\hline Septal defects & - & + & - \\
\hline Patent ductus arteriosus & - & + & - \\
\hline \multicolumn{4}{|l|}{ Dental features } \\
\hline Delayed/persistent/unerupted dentition & + & + & + \\
\hline Root radiculomegaly (secondary teeth) & ND & ND & ND \\
\hline Hypodontia (secondary teeth) & + & ND & ND \\
\hline Duplication/fusion (secondary teeth) & ND & ND & ND \\
\hline \multicolumn{4}{|l|}{ Skeletal features } \\
\hline Hammer toes (camptodactyly) & + & + & + \\
\hline Second-third toe syndactyly & + & + & + \\
\hline Broad halluces (big toe) & - & + & + \\
\hline Brachyphalangia of the fourth right toe & - & + & - \\
\hline Radioulnar synostosis & ND & ND & ND \\
\hline Lodosis/scoliosis/vertebral fusion & ND & ND & ND \\
\hline \multicolumn{4}{|l|}{ Other features } \\
\hline Mental retardation & - & - & + \\
\hline Hearing impairment & - & ND & ND \\
\hline Inguinal hernia & - & - & + \\
\hline
\end{tabular}

Abbreviation: ND, Not determined.

A plus (+) or minus (-) sign denotes the presence or absence of a particular physical feature.

$0.5 \mathrm{U}$ KOD FX polymerase (Toyobo). PCR were cycled 35 times at $98^{\circ} \mathrm{C}$ for $10 \mathrm{~s}, 68^{\circ} \mathrm{C}$ for $10 \mathrm{~min}$. PCR products electrophoresed in $1 \%$ agarose gel were stained with ethidium bromide and the aberrant band was extracted using QIAEXII Gel Extraction Kit (QIAGEN, Tokyo, Japan) and sequenced. Primer information is available on request.

\section{$\mathrm{X}$ inactivation study and haplotype analysis}

$\mathrm{X}$ inactivation pattern was studied using the human androgen receptor assay and fragile $\mathrm{X}$ mental retardation locus methylation assay as previously described. ${ }^{13-15}$ Briefly, genomic DNA of a patient (MC17), the parents (MC17a and MC17b), a control male and a control female was digested with two methylation-sensitive enzymes, HpaII and HhaI. PCR was performed with human androgen receptor assay primers (FAM-labeled ARf: 5'-CCAGAAT CTGTTCCAGAGCGTGC-3'; ARr: 5'-CTCTACGATGGGCTTGGGGAGAA $\left.\mathrm{C}-3^{\prime}\right)^{16}$ and fragile $\mathrm{X}$ mental retardation assay primers (FAM-labeled FMR 1f: 5'-AGCCCCGCACTTCCACCACCAGCTCCTCCA-3'; FMR1r: 5'-GCTCAGCT CCGTTTCGGTTTCACTTCCGGT- ${ }^{\prime}$ ). Fluorescent-labeled products were analyzed on an ABI PRISM 3100 or $3130 \times 1$ Genetic analyzer and GeneMapper Software version 4.0 (Applied Biosystems). One of affected females (MC18) was not analyzed because of insufficient amount of genomic DNA. According to published criteria, $\mathrm{X}$ inactivation ratios of $\leqslant 80: 20$ were considered random and ratios $>80: 20$ were considered skewed and ratios $>90: 10$ were considered highly skewed. ${ }^{16,17}$
X chromosome haplotype was analyzed using 12 microsatellite markers (DXS1060, DXS8051, DXS987, DXS1226, DXS1214, DXS1068, DXS993, DXS991, DXS986, DXS8055, DXS1047 and DXS1073). Fluorescent- labeled (either FAM, VIC or NED) forward primers and tailed reverse primers were purchased from Applied Biosystems. These markers were based on the Marshfield genetic map (http://research.marshfieldclinic.org/genetics). PCR was cycled 40 times at $94{ }^{\circ} \mathrm{C}$ for $30 \mathrm{~s}, 55^{\circ} \mathrm{C}$ for $30 \mathrm{~s}$ and $72^{\circ} \mathrm{C}$ for $30 \mathrm{~s}$ in $10 \mu \mathrm{l}$ volume containing $50 \mathrm{ng}$ DNA, $1 \times$ ExTaq buffer, $0.2 \mathrm{~mm}$ each dNTP, $0.4 \mu \mathrm{M}$ each primer and $0.1 \mathrm{U}$ ExTaq $\mathrm{HS}$ polymerase. Haplotypes were manually constructed.

\section{RESULTS}

We detected a BCOR mutation, c. 888delG in MC17, MC17b and MC18 (Figures 1d and e). The mutation may result in insertion of 80 new amino acids after the mutation site with a premature stop codon at position 377 (p.N297IfsX80). The mutation was completely co-segregated with OFCD phenotypes in this family (Figure 1a). Sequencing of the entire NHS coding region detected no pathological mutations in this family.

The Cytogenetics Whole-Genome $2.7 \mathrm{~m}$ array detected a total of 48 CNVs (12 duplications and 36 deletions) in any of the affected females. The CNVs, which encompassed exons, were 8 duplications and 13 deletions. Among them, two duplications and one deletion were shared with three affected females. The one deletion was present in the Database of Genomic Variants. Thus, the CNVs fulfilling the criteria for pathogenic $\mathrm{CNV}$ s were two interstitial duplications. The 740-kb duplication at Xp22.2-22.13 encompassed exons 2-18 of REPS2 (MIM ${ }^{\star} 300317$ ) and exons $1-3$ of NHS. The other $110-\mathrm{kb}$ duplication at Xp21.3 contained exon 2 of interleukin-1 receptor accessory protein-like 1 (IL1RAPL1) (MIM *300206) (Figure 2a). We were unable to examine the duplications by fluorescent in situ hybridization because only DNA samples were available. Instead, inverse PCR of self-ligated DNA with different sets of primers was able to amplify an expected fragment from normal NHS allele in all sets of primers, suggesting that the presence of one or more normal NHS alleles (Figures $2 \mathrm{~b}$ and $\mathrm{c}$ ). In addition, several attempts successfully cloned one of rearrangement junctions in relation to NHS (Figure 2c). This aberrant band showed that the sequences of intron 1 of IL1RAPL1 followed the sequences of intron 3 of NHS, suggesting that two duplications were tandemly connected (Figure 2d, upper cases). More complicatedly, 62-bp sequences of intron 3 of IL1RAPL1 with inverted orientation were inserted between intron 3 of NHS and intron 1 of IL1RAPL1 (Figure 2d, lower cases). The other possible breakpoints, which may result in disruption of NHS locus could not be determined regardless of rigorous attempts. Thus there seems to be two normal NHS alleles and an extra NHS allele, in which exons 1-3 of NHS was connected to exon 2 of ILIRAPL1. Copy number of the $B C O R$ gene was normal. In human androgen receptor assays, the mother (MC17b) was skewed pattern $(8 \%)$, whereas the elder sister (MC17) showed a random pattern (26\%). In fragile X mental retardation assays, the mother $(\mathrm{MC17b})$ and elder sister (MC17) showed a highly skewed pattern $(<1 \%)$ and a random pattern (48\%), respectively. X-chromosome haplotype analysis was able to separate all the alleles in this family (Supplementary Figure 1). The inactivated allele in the mother harbored the BCOR mutation and the NHS rearrangement.

\section{DISCUSSION}

BCOR functions as a corepressor of BCL-6, which is a POZ/zinc finger transcription repressor. ${ }^{4} \mathrm{BCOR}$ have three consecutive ankyrin motifs, an AF9 (ALL1 fused gene from chromosome 9) binding site and an NSPC1 (nervous system polycomb-1) binding site. ${ }^{4,18,19}$ Recently, the 


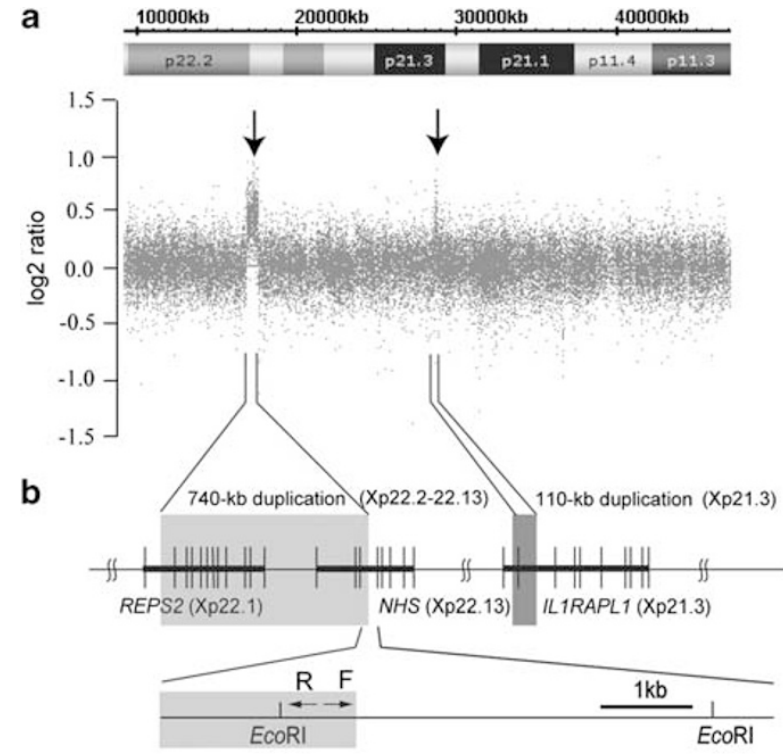

C

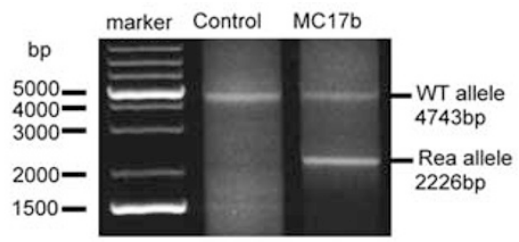

d

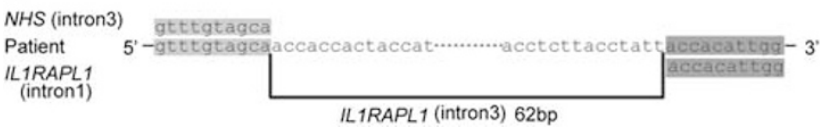

Figure 2 Two microduplications at Xp22.2-22.13 and Xp21.3. (a) Array profile of a part of chromosome $X$ in the elder sister (MC17). $x$ and $y$ axis show the genomic location from the $p$ telomere of chromosome $X$ (UCSC coordinates, May, 2006) (upper) and $\log _{2}$ (Signal ratio) values (lower), respectively. The 740-kb duplication at Xp22.2-22.13 and the 110-kb duplication at Xp21.3 are indicated by arrows. (b) Upper shows schematic representation of two duplicated regions (highlighted in gray). Three genes (REPS2, NHS and ILIRAPL1) are oriented in the same direction (from centromere to telomere). Lower indicates scheme of inverse PCR using selfligated DNA after digestion with EcoRI. Primers are shown by arrows ( $F$, forward; R, reverse). (c) Gel image of inverse PCR products performed as shown in (b). Upper and lower bands represent wild-type allele (WT allele) and rearranged allele (Rea allele), respectively. The DNA of both bands was extracted from agarose gel and sequenced. (d) Sequence of a Rea allele is shown. The top, middle and bottom indicate sequences of the intron 3 of NHS, rearranged junction and intron 1 of ILIRAPL1, respectively. The 62 nucleotides of intron 3 of ILIRAPL1 (lower cases) were inserted in an inverted orientation between intron 3 of NHS and intron 1 of ILIRAPLI. Matched sequences are highlighted with gray shadows.

minimal BCL-6 binding site was identified within residues 498-514, which is located in exon $4 .{ }^{20}$ In this study, a novel BCOR mutation, c.888delG (p.N297IfsX80) in exon 4, was found in this family. We assumed the mutant transcript with this mutation may undergo nonsense-mediated mRNA decay, but we could not confirm it as no living cells were available from the patients. A total of 31 mutations in BCOR were registered in Human Gene Mutation Database (http:// www.biobase-international.com/). All the mutations result in premature stop codons. Exon 4 harbors $40 \%$ of mutations (12/30), leading to truncated proteins lacking BCL-6, AF9 and NSPC1 binding sites, and ankyrin motifs if translated. Lack of BCL- 6 binding site could not explain the OFCD phenotypes because BCL-6-deficient mice did not show ocular, dental and skeletal phenotypes. ${ }^{21,22}$ Moreover, an OFCD mutant protein, that lacked ankyrin motifs and NSPC1 binding site, showed transcriptional repression activity similar to that of wild type. ${ }^{3}$ Thus, the c.888delG mutation in BCOR may be associated with loss of $B C O R$ transcripts through nonsense-mediated mRNA decay.

By genomic microarray, two microduplications were detected in MC17, MC18 and MC17b: one at Xp22.2-22.13 involving a part of REPS2 and NHS, and the other at Xp21.3 containing exon 2 of IL1RAPL1. Previously, complex duplication-triplication rearrangements of the NHS gene in a family with congenital cataract were reported, suggesting that genomic rearrangements of NHS are able to cause the X-linked cataract. ${ }^{8}$ This family did not show the typical features of NHS such as dental anomalies, dysmorphism and developmental delay, and congenital heart defects were diagnosed in four out of six affected males. The complex rearrangement consists of triplication embedded within a duplication region. The triplicated region includes the NHS genes except exon 1, and the entire SCML1 and RAI2 genes. Coccia et al. ${ }^{8}$ described that the additional phenotype of congenital heart defects observed in some affected males could be because of perturbed NHS gene transcription or increased dosage of the NHS, SCML1 or RAI2 genes. In our cases, two normal NHS alleles may exist in addition to an extra NHS allele, in which exons 1-3 of NHS was connected to individual exon 2 of IL1RAPL1, keeping the protein coding frame if properly spliced. Because the transcript from the extra NHS allele did not have polyA signal sequences, the allele is likely to produce no functional protein. Thus, the situation was totally different between our cases and Coccia's et al.'s ${ }^{8}$ cases.

Recently, Honda et al. ${ }^{23}$ reported two unrelated X-linked mental retardation Japanese families, which possessed the similar duplication found in our Korean family: the 737-kb duplication at Xp22.2, which contains a part of REPS 2 and NHS, and the $100-\mathrm{kb}$ duplication at Xp21.3, which contains a part of IL1RAPL1. In their report, fluorescent in situ hybridization analysis revealed that the clone RP11438J7, which entirely covered the duplication at Xp21.3, demonstrated two distinct signals at $\mathrm{Xp}$ in metaphases, suggesting that the duplication at Xp21.3 was inserted separately from the original site. The clone RP11-2K15 at Xp22.2, spanning the breakpoint of REPS2, showed one bright strong signal, suggesting that the duplication at Xp22.2 occurred in the proximity. Interestingly, the one of two signals of the RP11-438J7 was close to that of RP11-2K15 at Xp22.2, suggesting that the duplication involving IL1RAPL1 was inserted at Xp22.2. Their result is consistent with our data of the duplication breakpoint, in which the breakpoint in NHS was connected with the breakpoint of IL1RAPL1. Based on our experiences, high-density array experiments of $>500$ Japanese cases never showed such the genomic rearrangement involving NHS, implying that the rearrangement is very rare in Japanese population. It is noteworthy that congenital cataract and dental abnormalities were not pointed out in all the members (males and carrier females). ${ }^{23}$ Thus, it is unlikely that the genomic rearrangement involving NHS causes congenital cataract and dental abnormalities as found in our family.

IL1RAPL1 is a causative gene for X-linked mental retardation, ${ }^{24}$ and the microduplication at Xp21.3 containing exon 2 of IL1RAPL1 was suggested to cause MR in affected males. ${ }^{23}$ Most carrier females of IL1RAPL1 mutations and the carrier mother of the microduplication involving IL1RAPL1 showed normal intelligence. ${ }^{23-25}$ In this study, one of the three affected females (MC18) had learning difficulties at school, which could be mild presentation of MR. As $18 \%$ of patients with $B C O R$ mutations showed $\mathrm{MR},{ }^{6}$ it is reasonable that the BCOR mutation, rather than IL1RAPL1 rearrangement, causes mild MR in MC18. 
Skewed and random X-inactivation in the mother (MC17b) and the elder daughter (MC17) was confirmed, respectively, in this family. In OFCD, skewed X-inactivation with the preferential inactivation of the mutated allele were recognized in eight affected females (like the mother, $\mathrm{MC17b}$ ), suggesting that the $B C O R$ mutations may lead to a selective disadvantage in blood leukocytes. ${ }^{3,26}$ However, it has been reported that the $\mathrm{X}$-inactivation pattern in blood leukocytes are unable to determine the severity of disease phenotypes as X-inactivation pattern may vary among tissues. ${ }^{27}$ We suspect that $\mathrm{X}$-inactivation pattern is different between peripheral blood leukocytes and respective tissues associated with OFCD phenotype.

In conclusion, a new OFCD family is described with a novel $B C O R$ mutation. Clinical features overlap between OFCD and NHS, both of which belong to a spectrum of X-linked microphthalmia disorders. ${ }^{6}$ Our cases have a BCOR mutation and genomic rearrangements involving NHS, confusing us to address the genetic etiology in the family. However, the presence of bilateral 2nd-3rd toe syndactyly and septal heart defects, which is unique to OFCD, can lead us to the conclusion that the BCOR mutation is the major determinant for the phenotypes in this family. Careful examination of associated anomalies will be useful for genetic testing of X-linked microphthalmia disorders.

\section{ACKNOWLEDGEMENTS}

We thank the family members for their participation in this study. This work was supported by Research Grants from the Ministry of Health, Labour and Welfare (HS, N Miyake and N Matsumoto) and the Japan Science and Technology Agency (N Matsumoto) and Grant-in-Aid for Scientific Research from Japan Society for the Promotion of Science (N Matsumoto).

\section{Web Resources}

The URLs for data presented herein are as follows: UCSC Genome Browser, http:// genome.ucsc.edu/cgi-bin/hgGateway

1 Wilkie, A. O., Taylor, D., Scambler, P. J. \& Baraitser, M. Congenital cataract, microphthalmia and septal heart defect in two generations: a new syndrome? Clin. Dysmorphol. 2, 114-119 (1993).

2 Aalfs, C. M., Oosterwijk, J. C., van Schooneveld, M. J., Begeman, C. J., Wabeke, K. B. \& Hennekam, R. C. Cataracts, radiculomegaly, septal heart defects and hearing loss in two unrelated adult females with normal intelligence and similar facial appearance: confirmation of a syndrome? Clin. Dysmorphol. 5, 93-103 (1996).

3 Ng, D., Thakker, N., Corcoran, C. M., Donnai, D., Perveen, R., Schneider, A. et al. Oculofaciocardiodental and Lenz microphthalmia syndromes result from distinct classes of mutations in BCOR. Nat. Genet. 36, 411-416 (2004).

4 Huynh, K. D., Fischle, W., Verdin, E. \& Bardwell, V. J. BCoR, a novel corepressor involved in BCL-6 repression. Genes Dev. 14, 1810-1823 (2000).

5 Wamstad, J. A. \& Bardwell, V. J. Characterization of Bcor expression in mouse development. Gene Expr. Patterns 7, 550-557 (2007).

6 Hilton, E., Johnston, J., Whalen, S., Okamoto, N., Hatsukawa, Y., Nishio, J. et al. BCOR analysis in patients with OFCD and Lenz microphthalmia syndromes, mental retardation with ocular anomalies, and cardiac laterality defects. Eur. J. Hum. Genet. 17, 1325-1335 (2009).
7 Nance, W. E., Warburg, M., Bixler, D. \& Helveston, E. M. Congenital X-linked cataract, dental anomalies and brachymetacarpalia. Birth Defects Orig. Artic. Ser. 10, 285-291 (1974).

8 Coccia, M., Brooks, S. P., Webb, T. R., Christodoulou, K., Wozniak, I. O., Murday, V. et al. X-linked cataract and Nance-Horan syndrome are allelic disorders. Hum. Mol. Genet. 18, 2643-2655 (2009)

9 Lewis, R. A., Nussbaum, R. L. \& Stambolian, D. Mapping X-linked ophthalmic diseases. IV. Provisional assignment of the locus for X-linked congenital cataracts and microcornea (the Nance-Horan syndrome) to Xp22.2-p22.3. Ophthalmology. 97, 110-120; discussion 120-111 (1990).

10 Stambolian, D., Lewis, R. A., Buetow, K., Bond, A. \& Nussbaum, R. Nance-Horan syndrome: localization within the region Xp21.1-Xp22.3 by linkage analysis. Am. J. Hum. Genet. 47, 13-19 (1990).

11 Burdon, K. P., McKay, J. D., Sale, M. M., Russell-Eggitt, I. M., Mackey, D. A., Wirth, M. G. et al. Mutations in a novel gene, NHS, cause the pleiotropic effects of Nance-Horan syndrome, including severe congenital cataract, dental anomalies, and mental retardation. Am. J. Hum. Genet. 73, 1120-1130 (2003).

12 Miyamoto, T., Yu, Y. S., Sato, H., Hayashi, H., Sakugawa, N., Ishikawa, M. et al. Mutational analysis of the human MBX gene in four Korean families demonstrating microphthalmia with congenital cataract. Turk J. Pediatr. 49, 334-336 (2007).

13 Allen, R. C., Zoghbi, H. Y., Moseley, A. B., Rosenblatt, H. M. \& Belmont, J. W. Methylation of Hpall and Hhal sites near the polymorphic CAG repeat in the human androgen-receptor gene correlates with X chromosome inactivation. Am. J. Hum. Genet. 51, 1229-1239 (1992).

14 Carrel, L. \& Willard, H. F. An assay for X inactivation based on differential methylation at the fragile X locus, FMR1. Am. J. Med. Genet. 64, 27-30 (1996).

15 Nishimura-Tadaki, A., Wada, T., Bano, G., Gough, K., Warner, J., Kosho, T. et al. Breakpoint determination of $\mathrm{X}$; autosome balanced translocations in four patients with premature ovarian failure. J. Hum. Genet. 56, 156-160 (2011).

16 Kubota, T., Nonoyama, S., Tonoki, H., Masuno, M., Imaizumi, K., Kojima, M. et al. A new assay for the analysis of X-chromosome inactivation based on methylation-specific PCR. Hum. Genet. 104, 49-55 (1999).

17 Amos-Landgraf, J. M., Cottle, A., Plenge, R. M., Friez, M., Schwartz, C. E., Longshore, J. et al. X chromosome-inactivation patterns of 1,005 phenotypically unaffected females. Am. J. Hum. Genet. 79, 493-499 (2006).

18 Srinivasan, R. S., de Erkenez, A. C. \& Hemenway, C. S. The mixed lineage leukemia fusion partner AF9 binds specific isoforms of the BCL-6 corepressor. Oncogene 22 , 3395-3406 (2003)

19 Gearhart, M. D., Corcoran, C. M., Wamstad, J. A. \& Bardwell, V. J. Polycomb group and SCF ubiquitin ligases are found in a novel BCOR complex that is recruited to BCL6 targets. Mol. Cell Biol. 26, 6880-6889 (2006).

20 Ghetu, A. F., Corcoran, C. M., Cerchietti, L., Bardwell, V. J., Melnick, A. \& Prive, G. G. Structure of a BCOR corepressor peptide in complex with the BCL6 BTB domain dimer. Mol. Cell. 29, 384-391 (2008).

21 Ye, B. H., Cattoretti, G., Shen, Q., Zhang, J., Hawe, N., de Waard, R. et al. The BCL-6 proto-oncogene controls germinal-centre formation and Th2-type inflammation. Nat. Genet. 16, 161-170 (1997).

22 Dent, A. L., Shaffer, A. L., Yu, X., Allman, D. \& Staudt, L. M. Control of inflammation, cytokine expression, and germinal center formation by BCL-6. Science $\mathbf{2 7 6}, \mathbf{5 8 9 - 5 9 2}$ (1997).

23 Honda, S., Hayashi, S., Imoto, I., Toyama, J., Okazawa, H., Nakagawa, E. et al. Copynumber variations on the $\mathrm{X}$ chromosome in Japanese patients with mental retardation detected by array-based comparative genomic hybridization analysis. J. Hum. Genet. 55, 590-599 (2010).

24 Carrie, A., Jun, L., Bienvenu, T., Vinet, M. C., McDonell, N., Couvert, P. et al. A new member of the IL-1 receptor family highly expressed in hippocampus and involved in Xlinked mental retardation. Nat. Genet. 23, 25-31 (1999).

25 Nawara, M., Klapecki, J., Borg, K., Jurek, M., Moreno, S., Tryfon, J. et al. Novel mutation of ILIRAPL1 gene in a nonspecific X-linked mental retardation (MRX) family. Am. J. Med. Genet. A 146A, 3167-3172 (2008).

26 Hedera, P. \& Gorski, J. L. Oculo-facio-cardio-dental syndrome: skewed X chromosome inactivation in mother and daughter suggest $\mathrm{X}$-linked dominant Inheritance. Am. J. Med. Genet. A 123A, 261-266 (2003).

27 Bartnik, M., Derwinska, K., Gos, M., Obersztyn, E., Kolodziejska, K. E., Erez, A. et al. Early-onset seizures due to mosaic exonic deletions of CDKL5 in a male and two females. Genet. Med. 13, 447-452 (2011).

Supplementary Information accompanies the paper on Journal of Human Genetics website (http://www.nature.com/jhg) 